\title{
Erratum to: Meso-scale analysis on shear failure characteristics of asphalt-aggregate interface
}

\author{
Xin Qiu - Shanglin Xiao - Qing Yang • Yujie Wang • Feng Wang
}

Published online: 2 October 2017

(C) RILEM 2017

Erratum to: Materials and Structures (2017) 50:209 DOI 10.1617/s11527-017-1075-x

Due to an unfortunate turn of events, this article was published with an erroneous version of Fig. 3. The thickness of the steel plate should be $4(\mathrm{~mm})$ instead of $40(\mathrm{~mm})$. Please find in this erratum the correct version of Fig. 3 that should be regarded as final version by the reader.

The online version of the original article can be found under doi:10.1617/s11527-017-1075-x.

X. Qiu $(\bowtie) \cdot$ S. Xiao · Q. Yang · Y. Wang

College of Engineering, Zhejiang Normal University,

Jinhua 321004, China

e-mail: xqiu@zjnu.cn

S. Xiao

e-mail: zjnuxsl@gmail.com

Q. Yang

e-mail: yangq@zjnu.cn

Y. Wang

e-mail: wyj@zjnu.net

F. Wang

Department of Civil and Environmental Engineering,

Jackson State University, Jackson 39204, USA

e-mail: feng.wang@jsum.edu 
Fig. 3 Direct shear test apparatus (dimensions in $\mathrm{mm}$ )

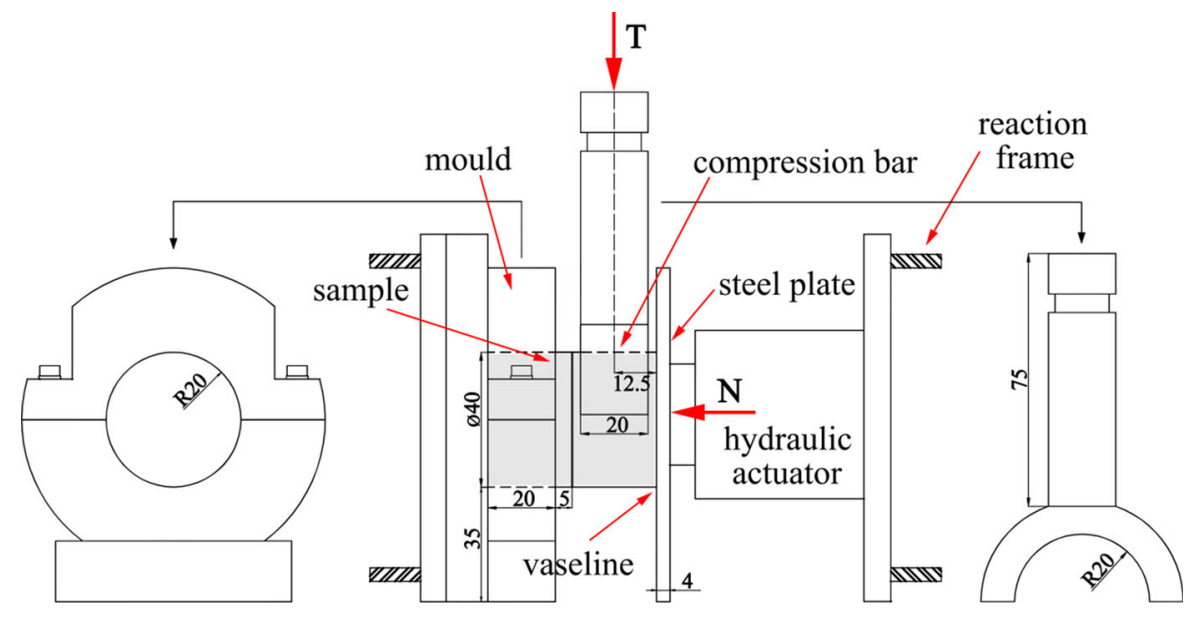

